\title{
Erratum to: Description of Endozoicomonas arenosclerae sp. nov. using a genomic taxonomy approach
}

\author{
Luciana R. Appolinario - Diogo A. Tschoeke - Cintia P. J. Rua • \\ Tainá Venas • Mariana E. Campeão • Gilda R. S. Amaral • \\ Luciana Leomil - Louisi de Oliveira - Verônica Viana Vieira • \\ Koko Otsuki · Jean Swings • Fabiano L. Thompson • Cristiane C. Thompson
}

Published online: 24 May 2016

(C) Springer International Publishing Switzerland 2016

\section{Erratum to: Antonie van Leeuwenhoek (2016) 109:431-438 \\ DOI 10.1007/s10482-016-0649-x}

Subsequent to the publication of the above paper, it has been noticed that some minor details, including one of the type strain culture collection codes, were missing from the original Abstract, Methods and protologue.

The last lines of the Abstract should read:

The species Endozoicomonas arenosclerae sp. nov. is proposed to harbour the new isolates. The type strain is CBAS $572^{\mathrm{T}}\left(=\mathrm{Ab} 112^{\mathrm{T}}=\mathrm{LMG} 29175^{\mathrm{T}}\right)$.

In Material and Methods the following amendment should be noted: The representative isolates $\mathrm{Ab} 112^{\mathrm{T}}$

The online version of the original article can be found under doi:10.1007/s10482-016-0649-x.

L. R. Appolinario - D. A. Tschoeke - T. Venas .

M. E. Campeão · G. R. S. Amaral · L. Leomil ·

L. de Oliveira $\cdot$ K. Otsuki $\cdot$ J. Swings .

F. L. Thompson · C. C. Thompson ( $₫)$

Instituto de Biologia, Universidade Federal do Rio de

Janeiro, Rio De Janeiro, RJ, Brazil

e-mail: thompsoncristiane@gmail.com

C. P. J. Rua

Instituto de Química de São Carlos, Universidade de São

Paulo, São Carlos, SP, Brazil

V. V. Vieira

Instituto Oswaldo Cruz (IOC), FIOCRUZ, Rio De Janeiro,

RJ, Brazil and Ab227_MC are deposited in the Bacteria Collection of Environmental and Health (CBAS) at Oswaldo Cruz Institute (IOC), FIOCRUZ (Rio de Janeiro, Brazil) (http://cbas.fiocruz.br/) under the accession numbers CBAS $572^{\mathrm{T}}$ and CBAS 573, and in the BCCM/LMG Bacteria Collection (http://bccm.belspo. be/) at Ghent University under the accession numbers LMG $29175^{\mathrm{T}}$ and LMG 29176, respectively.

The corrected protologue is given below.

\section{Formal description of Endozoicomonas arenosclerae sp. nov.}

Endozoicomonas arenosclerae (a.re.no.scle'rae. N.L. gen. n. arenosclerae of the sponge Arenosclera brasiliensis).

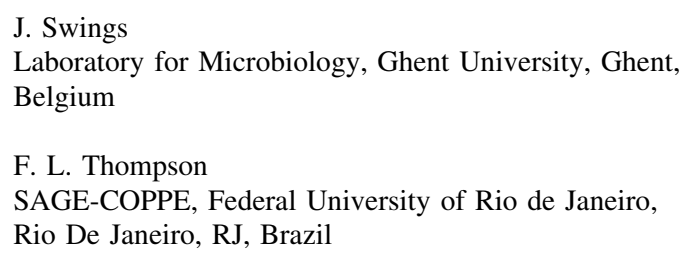


Cells are Gram-negative, aerobic, motile, $0.5-1.0 \mathrm{~lm}$ in diameter after incubation for $48 \mathrm{~h}$ at $30{ }^{\circ} \mathrm{C}$. Growth occurs at $12-35{ }^{\circ} \mathrm{C}$ in the presence of 2-4\% NaCl. Optimum growth occurs at $20-30{ }^{\circ} \mathrm{C}$ in the presence of $3 \% \mathrm{NaCl}$. Colonies are cream coloured, circular and convex with entire margins on Marine Agar. In API 20E, can utilise L-arginine and gelatin, but not 2-nitrophenyl- $\beta$-D-galactopyranoside, L-lysine, L-ornithine, trisodium citrate, sodium thiosulfate, L-tryptophan (TDA), L-tryptophan (IND), sodium pyruvate, D-glucose, D-mannitol, inositol, Dsorbitol, L-rhamnose, D-sucrose, D-melibiose, amygdalin and L-arabinose. Vitek analysis shows activity for phosphatase but not for adonitol, L-pyrrolidonylarylamidase, L-arabitol, D-cellobiose, $\beta$-galactosidase, $\mathrm{H}_{2} \mathrm{~S}$ production, glutamyl arylamidase, D-glucose, gamma-glutamyl-transferase, glucose, $\beta$-glucosidase, D-maltose, D-mannitol, D-mannose, $\beta$-xylosidase, $\beta$ alanine arylamidase, lipase, palatinose, tyrosine arylamidase, D-sorbitol, saccharose-sucrose, D-tagatose, trehalose, citrate (sodium), malonate, 5-keto-D-gluconate, L-lactate alkalinisation, $\alpha$-glucosidase, succinate alkalinisation, $\beta$ - $N$-acetyl-galactosaminidase, $\alpha$-galactosidase, glycine arylamidase, ornithine decar- boxylase, lysine decarboxylase, L-histidine assimilation, coumarate, $\beta$-glucuronidase, O-129 resistance, glu-gly-arg-arylamidase, L-malate assimilation, Ellman and L-lactate assimilation. In silico phenotypes predicted from genome sequences suggests strains are positive for alkaline phosphatase, $N$-acetyl- $\beta$-glucosaminidase, citric acid, succinic acid, L-alanine, Lserine, thymidine, glycerol, bacteriocin, siderophore and resorcinol and negative for C8 esterase, L-fucose, uridine and ectoine. The type strain has a DNA G+C content of $47.6 \mathrm{~mol} \%$.

The type strain CBAS $572^{\mathrm{T}}\left(=\mathrm{Ab} 112^{\mathrm{T}}=\mathrm{LMG}\right.$ $29175^{\mathrm{T}}$ ) was isolated from the marine sponge Arenosclera brasiliensis.

\section{Nucleotide sequence accession numbers}

The Whole Genome Shotgun Projects for E. arenosclerae $\mathrm{CBAS} 572^{\mathrm{T}}\left(=\mathrm{Ab} 112^{\mathrm{T}}\right)$ and E. arenosclerae $\mathrm{CBAS}$ 573 (= Ab227_MC) have been deposited in DDBJ/ EMBL/GenBank under accession numbers LASA 010000000 and LASB010000000, respectively. 